\title{
Automated Defect Inspection Algorithm Incorporated Spectral-Domain Optical Coherence Tomography for Optical Polarizing Thin Films
}

\author{
Byeonggyu Jeon, Youngmin Han, Ruchire Eranga Wijesinghe, Mansik Jeon, Jeehyun Kim
}

\begin{abstract}
Optical polarizing thin film is an optical filter enables light waves of a specific polarization pass through while blocking light waves of other polarizations. Optical polarizing thin films control the brightness of back-light unit for LCD (liquid crystal display) panel, which is essential to produce LCD modules. Defect inspection of polarizing thin films is an important feature during the manufacturing process that is helpful to improve the product quality. In the current study, an automated defect inspection algorithm is introduced and incorporated with a well-known non-destructive and non-contact optical inspection method called spectral domain optical coherence tomography (SD-OCT) to pre-identify defective sub-surface as well as top-surface locations of optical polarizing thin films Polarizing thin films employed in this study consist of 6 layers. The tomographic information, layer information, and defective locations were sufficiently identified through the SD-OCT system owing high-axial resolution. The acquired results indicate the possible application of the proposed system in optical polarizing thin films for the quality assurance.
\end{abstract}

Keywords: SD-OCT, optical polarizing thin film, defect inspection, optical inspection.

\section{INTRODUCTION}

Liquid crystal displays (LCDs) are broadly used in electronic products such as computers and smartphones. The competitiveness in the electronics are affected by the display quality and the components of the display must be verified. . LCD consists of back-light unit, polarizing film, liquid

Revised Manuscript Received on February 05, 2020.

* Correspondence Author

Youngmin Han, Department of Nuclear Energy Convergence, Kyungil University, Gyeongsan-si, Gyeongbuk, Korea, hanym116@gmail.com

Byeonggyu Jeon, School of Electronics Engineering, College of IT Engineering, Kyungpook National University, 80 Daehak-ro, Buk-gu, Daegu 41566, Korea, xe1700@gmail.com

Ruchire Eranga Wijesinghe, Department of Biomedical Engineering, College of Engineering, Kyungil University, Gyeongsan 38428, Korea, eranga@kiu.kr

Mansik Jeon*, School of Electronics Engineering, College of IT Engineering, Kyungpook National University, 80 Daehak-ro, Buk-gu, Daegu 41566, Korea, msjeon@knu.ac.kr

Jeehyun Kim, School of Electronics Engineering, College of IT Engineering, Kyungpook National University, 80 Daehak-ro, Buk-gu, Daegu 41566, Korea, jeehk@knu.ac.kr

(C) The Authors. Published by Blue Eyes Intelligence Engineering and Sciences Publication (BEIESP). This is an open access article under the CC BY-NC-ND license (http://creativecommons.org/licenses/by-nc-nd/4.0/) crystal and a colour filter. The light emitted from the back-light unit is uniformly vibrating in all directions. However, the polarizing thin film transmits only the incident light vibrating in the same direction as the polarization axis of the thin film and absorbs or reflects other light beams, which converts non-polarized light into polarized light in a particular direction $[1,2]$. When the polarized light passes through the LCD panel, the brightness of the pixel is adjusted by the array direction of each pixel in an electrical way. However, various structural defects inevitably occur in the polarizing thin films during the manufacturing process. Due to aforementioned defective locations, fundamental performance of the polarizing thin film cannot be obtained, while functions mentioned above can't be performed normally. Numerous inspection techniques have gained an enormous attention in assuring the quality of industrial products. Quality inspection of industrial products have been mostly succeeded using non-destructive and non-contact techniques incorporated with automated image processing and analysis. Visual inspection methods, machine vision with a charge-coupled device (CCD) camera-based inspection or electronic inspection have been frequently employed for the quality inspection in optical thin film industry [3, 4]. Since these techniques provide information of top surface information, highly penetrating $\mathrm{X}$-ray and computed tomographic techniques were considered for sub-surface, but none of them provides depth resolution at the micro-meter scale $[5,6]$. Since optical polarizing thin films based products are composed of several optical thin films, an optical inspection method with high depth-resolved resolution can be an ideal solution. Optical coherence tomography (OCT) is a broadband laser based non-destructive inspection method, which was primarily developed for medical imaging applications [7, 8]. The exceptional depth resolved and spatial resolution of this medical imaging technique has led it path towards industrial material inspections $[9,10]$. With the development of novel OCT systems, the diversity of OCT applications has been expanded from the biological domain to industrial investigations. OCT has played an important role in the accurate identification of defects in various industrial fields, such as touch-screen panel (TSP) inspection, verification of pearls, defect tracking of optical lenses, micro-meter range defect inspection of industrial resins, and identification of counterfeit notes [11-13]. 
It is necessary to have a high resolution to inspect even small defects in the polarizing films. Moreover, the polarizing film consists of several layers and tomographic information must be obtained to identify the defects lay within sub-surface layers.

Thus, OCT can be an ideal solution for the defect inspection of optical polarizing thin films.

In this study, OCT image intensity based automated defect tracking algorithm was developed to pre-identify defects of the optical polarizing thin film specimens. The developed imaging system is capable of detecting defective locations with a magnitude of micro-meter range in real-time due to high-resolution and high image acquisition speed. In LCD manufacturing process, fault detection is highly essential at an early stage so that the quality assurance of the final product can be maintained. The developed algorithm incorporated OCT provides cross-sectional information with high accuracy, and therefore, this study describes how OCT can be beneficial to enhance fault detection in the manufacturing process of LCD panels.

\section{MATERIALS AND METHODS}

\section{A. Preparation of the polarizing thin film samples}

The sub-surface layer information of the fabricated polarizing film is illustrated in Figure 1 schematic diagram. From the top surface, polarizing film consists of multiple layers, such as protective film, tri-acetyl cellulose (TAC) film, polyvinyl alcohol (PVA) layer, TAC film, pressure sensitive adhesive (PSA) and release film. External damages are prevented through the protective film, while TAC film is used to protect the PVA layer due to its high light transmittance, provide a high thermal stability, and high uniformity characteristics. PVA layer is the key part of the film that polarizes the light. PSA is a bonding material that attaches polarizing film to LCD. The release film is removed during the LCD production process. Therefore, the actual inspection area was set in four layers from a protective film to TAC film. The total thickness of the polarizing film is approximately $200 \mu \mathrm{m}$.

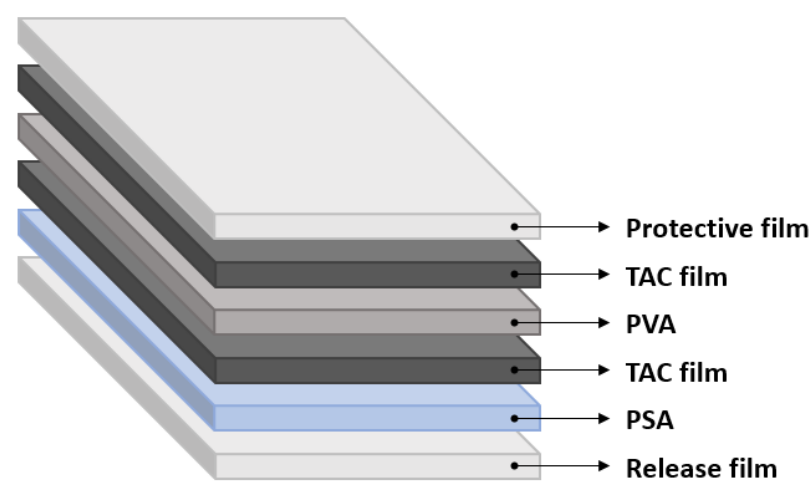

Fig. 1.The structure of optical polarizing thin film. The abbreviations are TAC: tri-acetyl cellulose. PVA: polyvinyl alcohol. PSA: pressure sensitive adhesive.

\section{B. SD-OCT system configuration}

Figure 2 depicts the schematic diagram of the laboratory customized SD-OCT system for the inspection of optical polarizing thin films. A super-luminescent diode (SLD 371-HP3, Superlum, Ireland) was used as the broadband laser source with a center wavelength of $835 \mathrm{~nm}$ and a full-width at half-maximum bandwidth of $50 \mathrm{~nm}$. The typical output power of the laser was $29.6 \mathrm{~mW}$, and the incident power on the sample after optical losses was 12.55 $\mathrm{mW}$. The utilized scan range for the samples was $5 \mathrm{~mm} \times 5$ $\mathrm{mm}$, which sufficiently scanned the entire region of interest. The laser beam of the source was split into sample and reference arms through a 50:50 fiber coupler. The reflected light form reference and sample arm was interfered at fiber coupler and detected by CCD line scan camera (spL-140km, Basler, Germany) with 4096-pixels and 70,000 lines/s effective line rate in the integrated optical spectrometer. The axial and transverse resolutions (in air) were measured as 7 and $25 \mu \mathrm{m}$, respectively. In real-time defect inspection environment, the speed of signal and image processing, such as fast Fourier transformation (FFT), logarithmic scaling and enface imaging are important factors. Thus, parallel computing platform using compute unified device architecture (CUDA) was employed, which allows the large amounts of operations simultaneously. Detail specifications of the system is reported elsewhere [14].

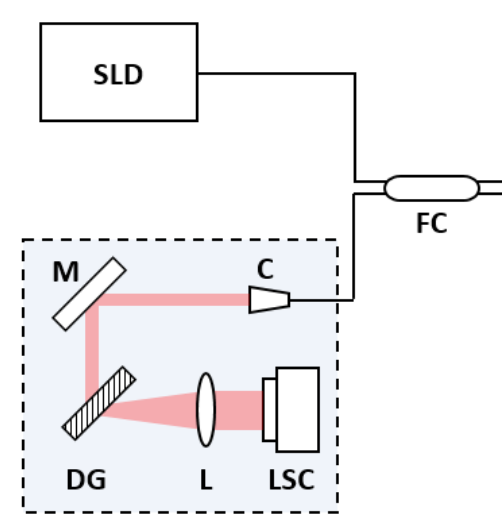

Spectrometer

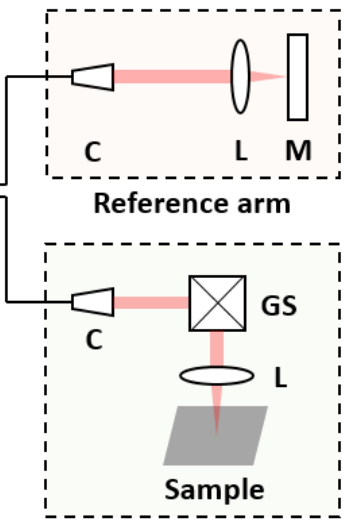

Sample arm
Fig. 2.Schematic diagram of SD-OCT. C: collimator. DG: diffraction grating. GS: galvanometer scanner. L: lens. LSC: line scan camera. M: mirror. SLD: super-luminescent diode.

\section{Defect inspection algorithm}

The graphical illustration of the automated defect inspection algorithm is shown in Figure 3 along with the cross-sectional OCT images obtained using the laboratory customized SD-OCT system. The dimension of each OCT image size was 1000 (transverse) x 500(depth), and 1000 cross-sectional images per sample were acquired to obtain volume renders. In the developed algorithm, preprocessing of the opening filter and binarization were performed initially. This opening filter operates dilation and erosion imaging processes, and noise in the images can be removed, while maintaining the shape of the polarizing film. Binarization is an imaging filter that sets a specific threshold value. Hence, this filter shows the polarizing film and noise with a clear visualization. In the next process, each layer of the films is separated. The averaged A-line (axial line illustrated as vertical red dashed line) was obtained by profiling the B-scan image horizontally. This averaged profile contains the information about the interface of the polarizing film layer. 
Therefore, the inspection areas are determined between the interfaces, and it is corresponded to the region of interest (ROI) of sub-surfaces. In each ROI layer, the pixel intensities are averaged in the vertical direction to create an enface image. This averaging process is performed in every cross-sectional image, and the enface images of every layers are constructed. The presence of defects can be determined from the enface images using the flood-fill algorithm.
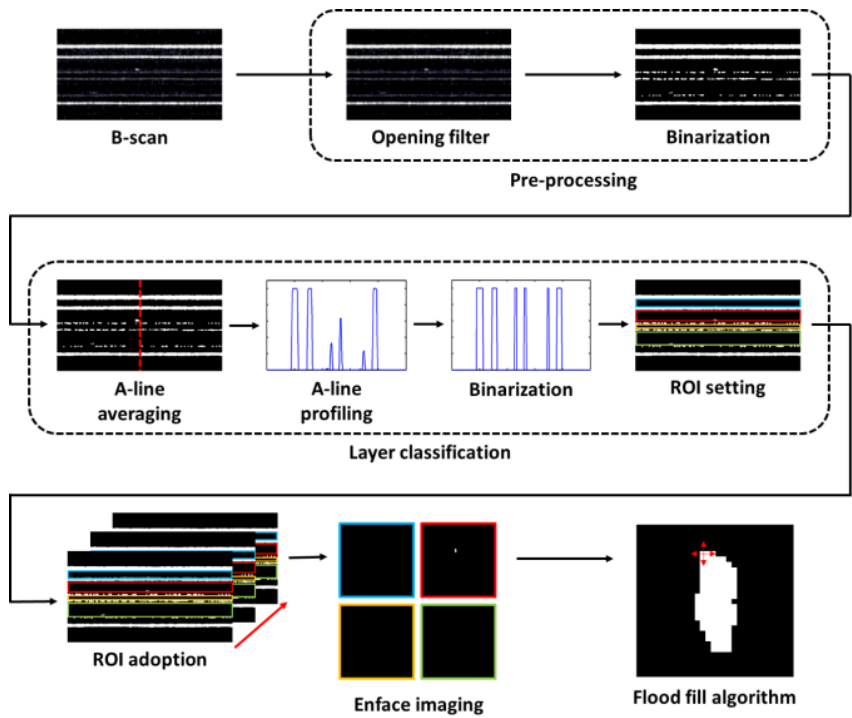

Fig. 3.Flowchart of automatic defect inspection algorithm.

\section{RESULTS AND DISCUSSION}

A. OCT cross-sectional and volumetric image assessments

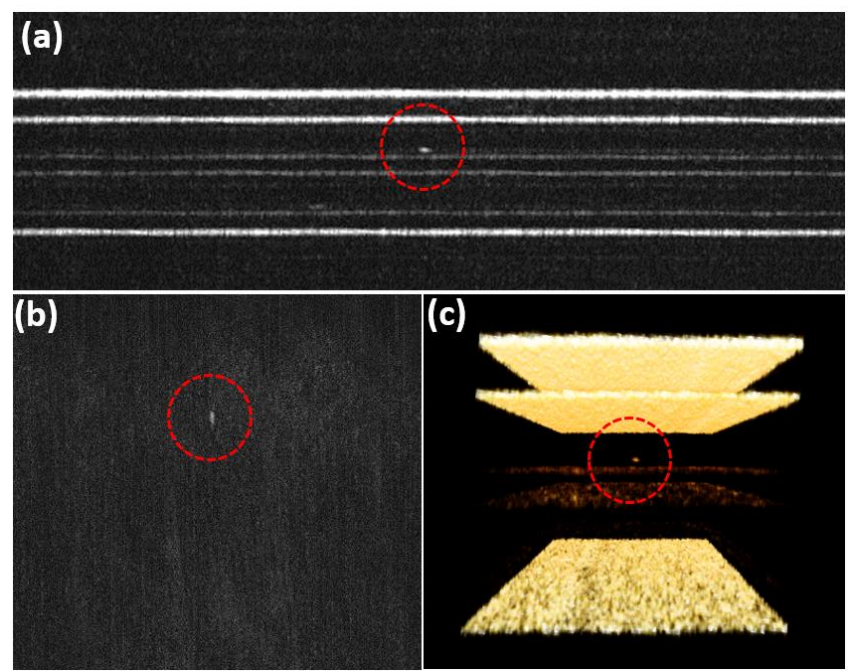

Fig. 4. OCT images of polarizing film. (a) B-scan image. (b) TAC enface image. (c) 3D OCT image.

Figure 4 illustrates the cross-sectional and volumetric results of optical polarizing thin film sample acquired using the developed SD-OCT system. Figure 4 (a) depicts the cross-sectional image of the examined polarizing thin film layer with a defect lay between layers. The defective location is indicated with a red color dotted circle. The sub-surface layer information described in Figure 1 is clearly revealed in Fig. 4 (a), and the cross-sectional image emphasizes the thickness of each layer and the distance between each layer.
Although the sample has multiple layers, the region of interest (defective location) can be identified as the TAC film layer. Thus, the focus is matched to TAC layer for a better visualization. Figure 4(b) shows the enface image, while figure 4 (c) is the volumetric representation of the region of interest. Hence, figure 4(c) illustrates the three dimensional magnitudes of the identified defect. One defect in the TAC film is visible in this image (under the protective film). Figure 4 (b) shows the enface image of a TAC film with a defect. Figure 4 (c) shows a three-dimensional OCT image of the film. This image shows the overall structure and defects of the polarizing film.

\section{B. Depth profile based defect assessment}
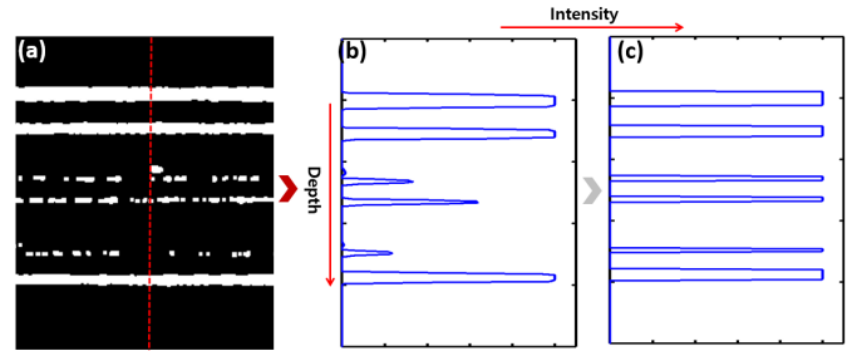

Fig. 5.Intensity profile along vertical line for layer classification.

Figure 5 depicts the OCT image intensity based depth dependent layer classification of the examined polarizing thin film sample. A-lines were acquired from the entire lateral pixel range to obtain a representative depth profile for the entire cross-section. Next, all the acquired A-lines were summed up and averaged to enhance the precision. Figure 5(a) shows the OCT image with a representative A-line, where figure 5(b) and (c) are the corresponding A-line depth profiles. Since the intensity profile also contains defect or noise signals, the binarization process is required.
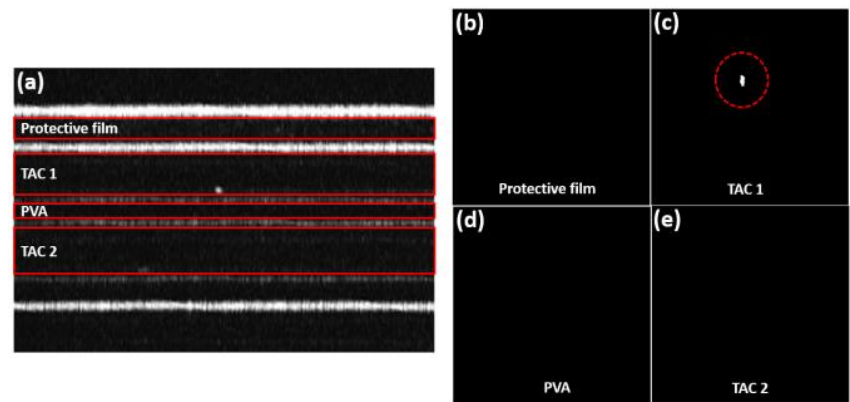

Fig. 6. Each layer of polarizing film and enface images.

The region of interest is decided on this binarized Intensity profile. Figure 6(a) illustrates the sub-surface layers of the polarizing thin film sample, such as protective film, TAC, PVA, and TAC layer, respectively from top to bottom. The release film is removed as it was removed during the manufacturing process. This ROI decision process is applied to every cross-sectional image, and each image has its own regions of interest. Figure 6 (b e) shows enface representation exhibiting four regions of interest of each sub-surface layer. Each enface image consists of 1000 cross-sectional images and its ROI layers, and the magnitude was 1000 (transverse) x 1000 (frames). 
The enface visualization simply determines the exact location of the defective particle as shown in figure 6(c). Hence, the generated enface images simply enable the identification of the exact defective location along with the exact layer information.

\section{CONCLUSION}

Development of a non-destructive inspection modality for the pre-identification of defective particles in optical polarizing thin film samples is introduced. High-resolution SD-OCT system was employed as the optical inspection modality to identify micro-meter range defective particles. Owing to sufficient depth penetration and the resolution of the system, sub-surface layer information and the defective particles were clearly identified. To enhance the applicability of the proposed method in industrial sector and achieve a high productivity, automated layer and defect detection algorithm was customized and incorporated with inspection modality. The layer detection algorithm performed successfully based on the pixel intensities and image filters. The enface images were generated based on the detected layers and the defects were detected using flood-fill algorithm. According to the result, it is expected to utilize this setup to test optical polarizing thin films in the early stage of the manufacturing process and this method can be well-utilized to remove the defective components rapidly. As a result, it is expected to create the quality assurance and industrial value using OCT and machine vision systems. In the future, it is needed to verify the detection.

\section{ACKNOWLEDGMENT}

This work was supported by the National Research Foundation of Korea (NRF) grant funded by the Korea government. (MSIT) (2019R1G1A1092172).

\section{REFERENCES}

1. Q. H. Wu, Birefringent thin films and polarizing elements: World Scientific, 1998.

2. I. Hodgkinson and Q. H. Wu, "Birefringent thin-film polarizers for use at normal incidence and with planar technologies," Applied physics letters, vol. 74, pp. 1794-1796, 1999.

3. R. M. Ostroff, D. Hopkins, A. B. Haeberli, W. Baouchi, and B. Polisky, "Thin film biosensor for rapid visual detection of nucleic acid targets," Clinical chemistry, vol. 45, pp. 1659-1664, 1999.

4. N.-V. Ngo, G. A. Porter, and Q.-C. Hsu, "Development of a Color Object Classification and Measurement System Using Machine Vision," Sensors and Materials, vol. 31, 2019.

5. A. Townsend, L. Pagani, L. Blunt, P. J. Scott, and X. Jiang, "Factors affecting the accuracy of areal surface texture data extraction from X-ray CT," CIRP Annals, vol. 66, pp. 547-550, 2017.

6. H. E. Martz, C. M. Logan, D. J. Schneberk, and P. J. Shull, X-ray Imaging: fundamentals, industrial techniques and applications: CRC Press, 2016.

7. E. A. Swanson, J. A. Izatt, M. R. Hee, D. Huang, C. Lin, J. Schuman, C. Puliafito, and J. G. Fujimoto, "In vivo retinal imaging by optical coherence tomography," Optics letters, vol. 18, pp. 1864-1866, 1993.

8. J. Welzel, "Optical coherence tomography in dermatology: a review," Skin Research and Technology: Review article, vol. 7, pp. 1-9, 2001.

9. T. Prykäri, J. Czajkowski, E. Alarousu, and R. Myllylä, "Optical coherence tomography as an accurate inspection and quality evaluation technique in paper industry," Optical Review, vol. 17, pp. 218-222, 2010.

10. R. Su, M. Kirillin, E. W. Chang, E. Sergeeva, S. H. Yun, and L. Mattsson, "Perspectives of mid-infrared optical coherence tomography for inspection and micrometrology of industrial ceramics," Optics express, vol. 22, pp. 15804-15819, 2014.
11. R. E. Wijesinghe, K. Park, Y. Jung, P. Kim, M. Jeon, and J. Kim, "Industrial resin inspection for display production using automated fluid-inspection based on multimodal optical detection techniques," Optics and Lasers in Engineering, vol. 96, pp. 75-82, 2017.

12. M. F. Shirazi, K. Park, R. E. Wijesinghe, H. Jeong, S. Han, P. Kim, M. Jeon, and J. Kim, "Fast industrial inspection of optical thin film using optical coherence tomography," Sensors, vol. 16, p. 1598, 2016.

13. M. F. Shirazi, R. E. Wijesinghe, N. K. Ravichandran, P. Kim, M. Jeon, and J. Kim, "Quality assessment of the optical thin films using line field spectral domain optical coherence tomography," Optics and Lasers in Engineering, vol. 110, pp. 47-53, 2018.

14. N. H. Cho, K. Park, J.-Y. Kim, Y. Jung, and J. Kim, "Quantitative assessment of touch-screen panel by nondestructive inspection with three-dimensional real-time display optical coherence tomography," Optics and Lasers in Engineering, vol. 68, pp. 50-57, 2015.

\section{AUTHORS PROFILE}

Byeonggyu Jeon received the B.E. degree in electronics engineering from Kyungpook National University, Daegu, South Korea, in 2018, where he is currently a M.Sc. Researcher with the Electronics Engineering Department. His research interests include development of optical system for medical application, photoacoustic tomography, and development and applications for spectral domain optical coherence tomography.

Youngmin Han received his B.E degree in mechanical engineering from Tohoku University, Sendai, Japan in 2012. He completed his M.Sc. in power engineering from Technical University of Munich, Munich, Germany. His research interests is in the development of advanced materials, including tungsten metals and molten salts, for nuclear fusion reactors using optical techniques and image processing. He is currently an Assistant Professor with the Department of Nuclear Energy Convergence, Kyungil University.

Ruchire Eranga Wijesinghe received the B.Sc. and Ph.D. degrees in electronics engineering from Kyungpook National University, Daegu, South Korea, in 2012 and 2018, respectively. He is currently an Assistant Professor with the Department of Biomedical Engineering, Kyungil University. His research interests include development of high-resolution novel biological and biomedical imaging techniques, including optical coherence tomography and microscopy for clinical utility.

Mansik Jeon received the Ph.D. degree in electronics engineering from Kyungpook National University, Daegu, South Korea, in 2011, where he is currently an Assistant Professor with the School of Electronics Engineering. His research interests include development of nonionizing and noninvasive novel biomedical imaging techniques, including photoacoustic tomography, photoacoustic microscopy, optical coherence tomography, ultrasonic imaging, handheld scanner, and their clinical applications.

Jeehyun Kim received the Ph.D. degree in biomedical engineering from the University of Texas at Austin, USA, in 2004. He was a Postdoctora Researcher with the Beckman Laser Institute, University of California at Irvine. He is currently a Professor with Kyungpook National University, Daegu, South Korea. His research interests include biomedical imaging and sensing, neuroscience studies using multiphoton microscopy, photoacoustic imaging, and other novel applications of sensors. 